\title{
Enhanced Stability Lithium-ion Battery based on Optimized Graphene/Si Nano Composite by Templated Assembly
}

Long Liu, $\uparrow$ Xinxi Li, ${ }^{*} \uparrow$ Guoqing Zhang, $\uparrow$ Zengyao Zhang, $\dagger$ Chenhui Fang, $\dagger$ Hong Ma, $\uparrow$ Wen Luo, *† and Zhongyun Liu $\ddagger$

$\dagger$ School of Materials and Energy, Guangdong University of Technology, Guangzhou 51006, PR China

$\$$ School of Chemical and Biomolecular Engineering, Georgia Institute of Technology, 311 Ferst Dr. NW Atlanta, GA 30332,USA

\section{Corresponding Author}

*E-mail: pkdlxx@gdut.edu.cn

wenluo@gdut.edu.cn 


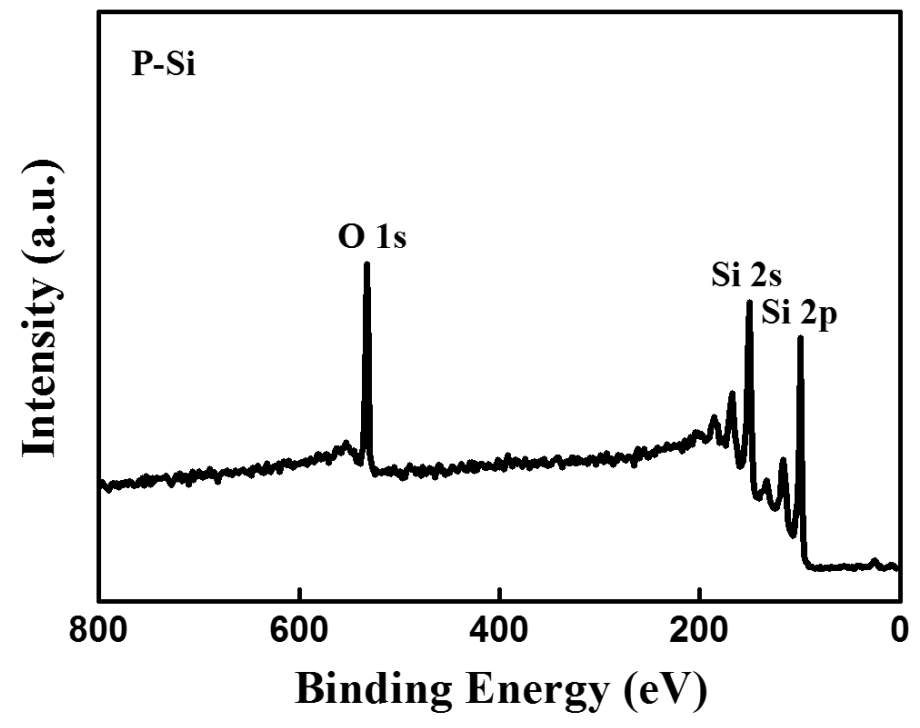

Figure S1. XPS spectra of P-Si.

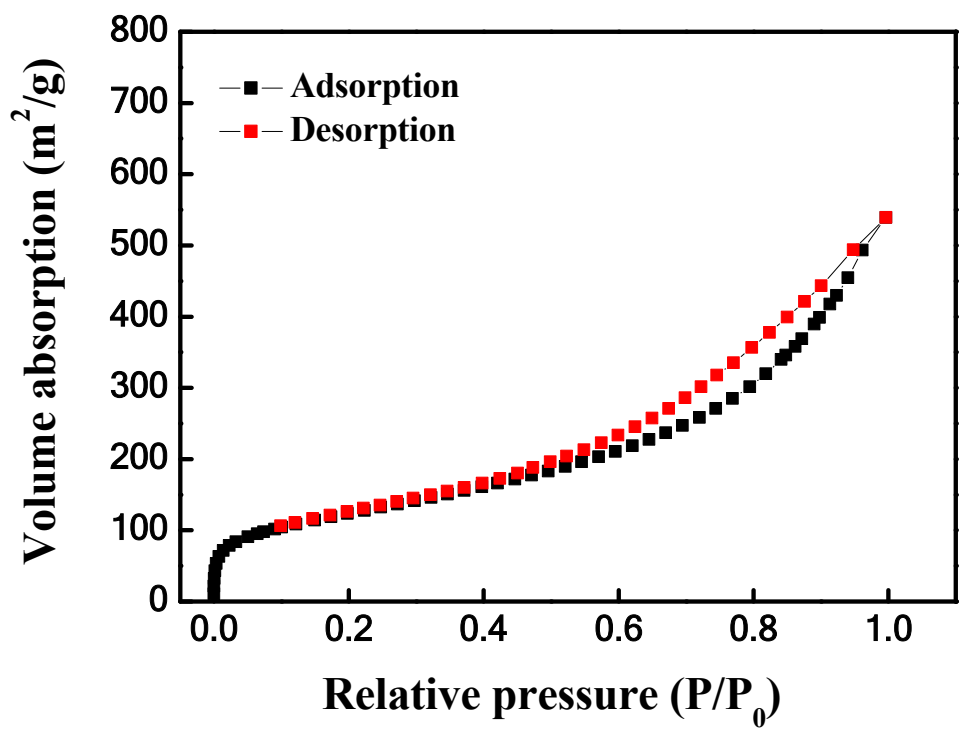

Figure S2. The adsorption/desorption of 10RGO/Si-600.
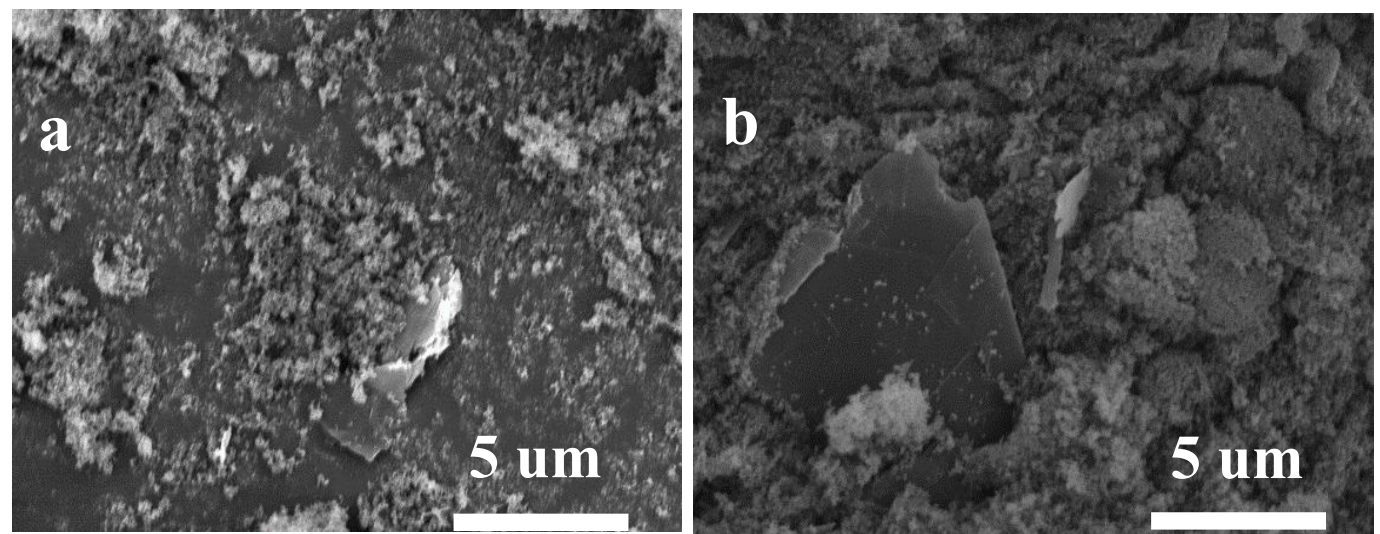
Figure S3. SEM images of (a) 5RGO/Si and (b) $15 \mathrm{RGO} / \mathrm{Si}$.

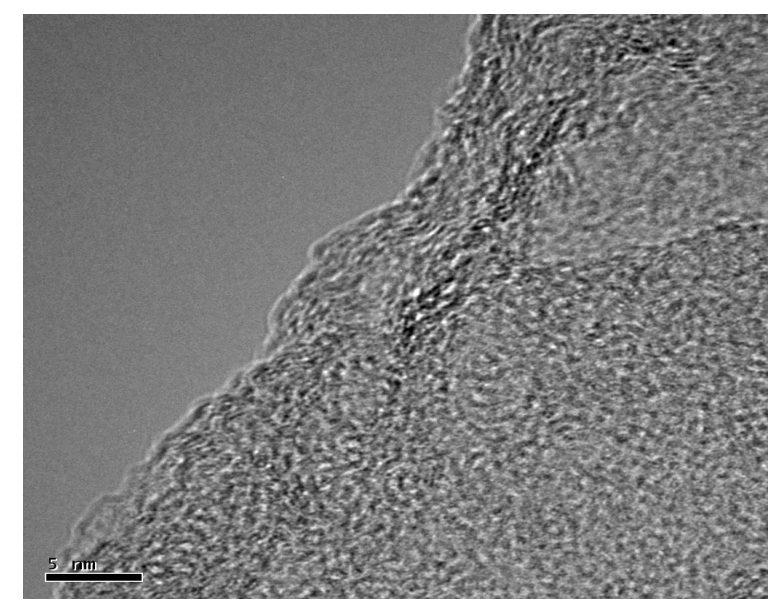

Figure S4. TEM image of graphene nanosheet with high resolution.
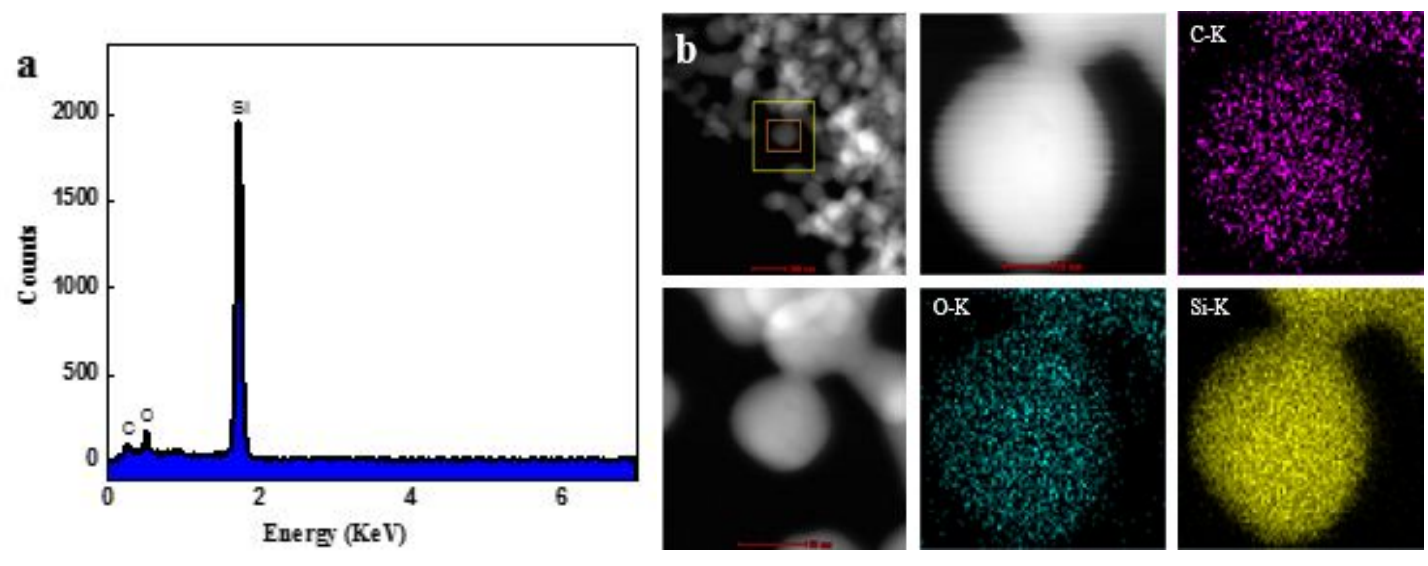

Figure S5. (a) EDS of the 10RGO/Si-600 composite and (b) the mapping images of 
each element.

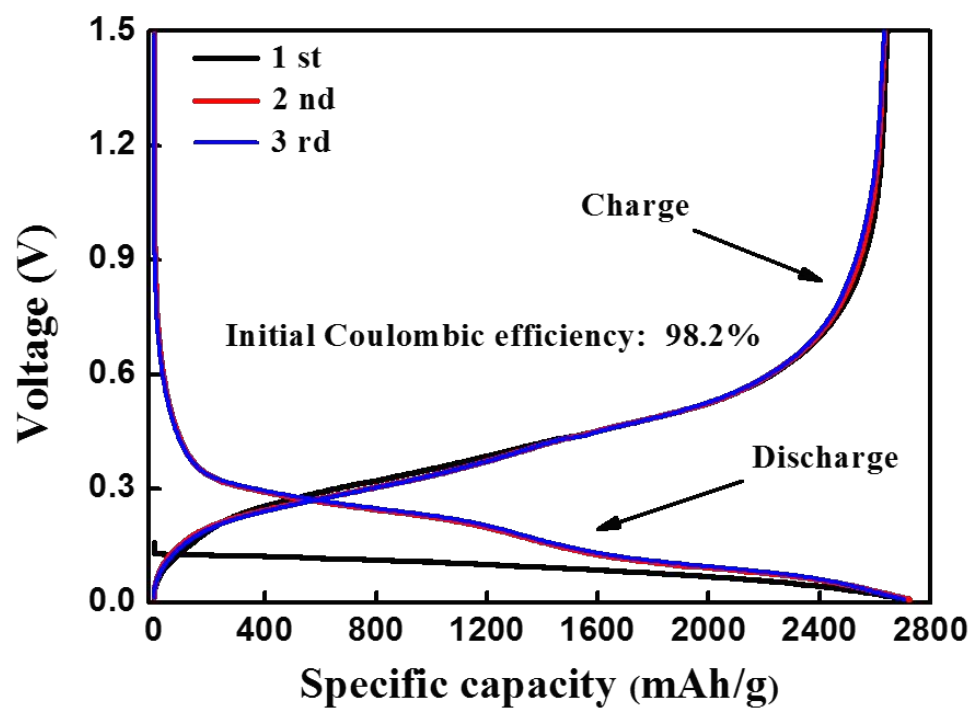

Figure S6. The first charge-discharge capacity of $10 \mathrm{RGO} / \mathrm{Si}$ at $0.1 \mathrm{~A} / \mathrm{g}$. 


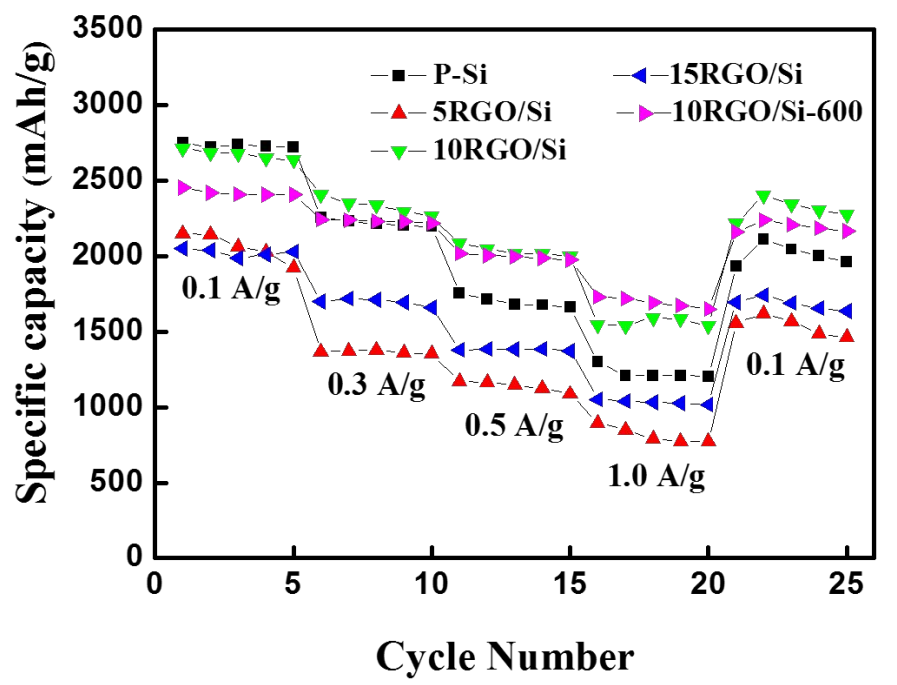

Figure S7. Rate performance of $\mathrm{P}-\mathrm{Si}$ and $\mathrm{RGO} / \mathrm{Si}$ composites at various current densities from 0.1 to $1.0 \mathrm{~A} / \mathrm{g}$.

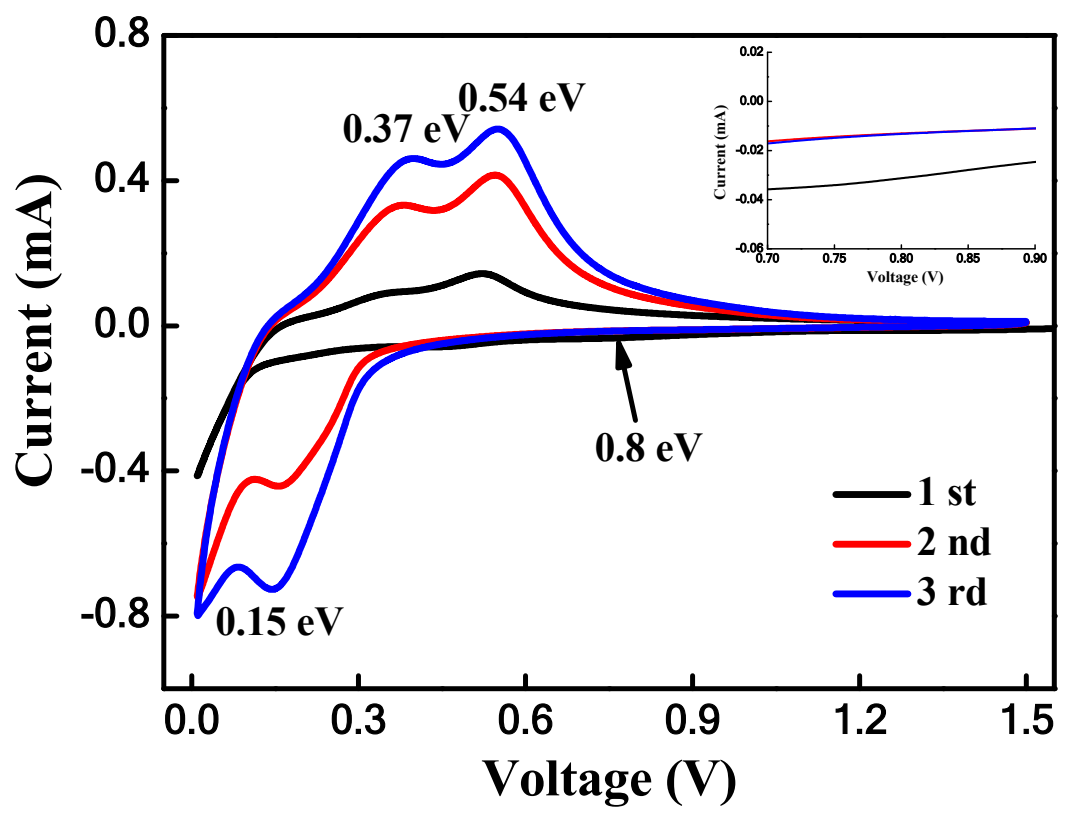

Figure S8. The CV curves of P-Si electrode at the first three cycles with the inset enlarged view. 


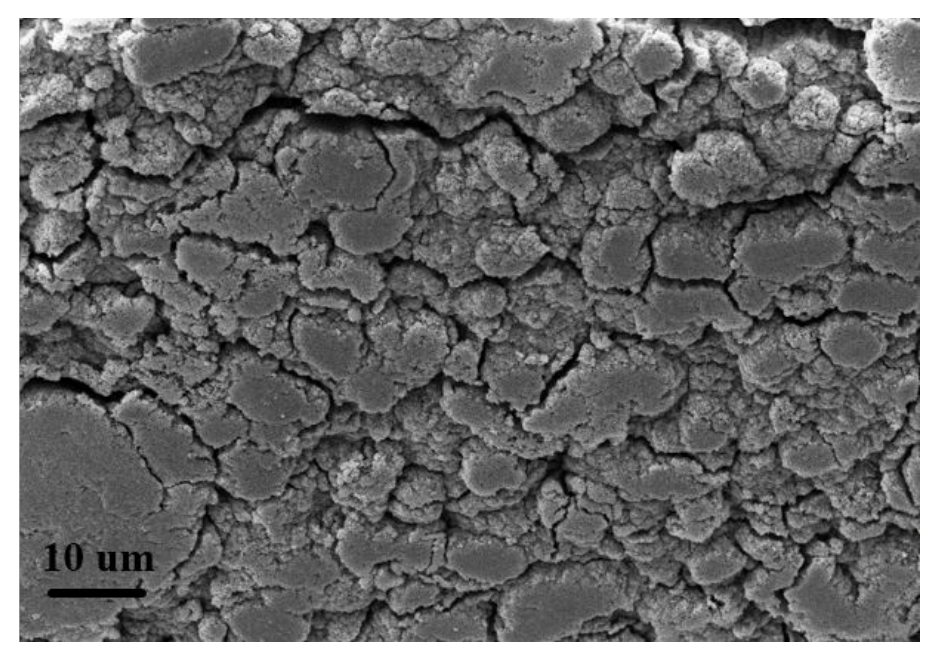

Figure S9. SEM images of the P-Si after 100 charge/discharge cycles at $0.1 \mathrm{~A} / \mathrm{g}$.

Table S1. The BET data of P-Si, 10RGO/Si and 10RGO/Si-600 samples.

\begin{tabular}{cccc}
\hline Sample & P-Si & 10RGO/Si & 10RGO/Si-600 \\
\hline $\operatorname{BET}\left(\mathrm{m}^{2} / \mathrm{g}\right)$ & 30 & 482 & 539 \\
\hline
\end{tabular}

Table S2. The EDS analysis of each element

\begin{tabular}{ccc}
\hline Element & Weight (\%) & Atomic (\%) \\
\hline C (K) & 1.96 & 4.33 \\
O (K) & 4.77 & 7.89 \\
Si (K) & 93.25 & 87.77 \\
Totals & 100.00 & \\
\hline
\end{tabular}


Table S3. The initial charge-discharge capacities and corresponding coulombic efficiency of the P-Si and RGO/Si at a constant current density of $0.1 \mathrm{~A} / \mathrm{g}$.

\begin{tabular}{cccc}
\hline Samples & $\begin{array}{c}\text { Initial Discharge } \\
\text { capacity }(\mathbf{m A h} / \mathbf{g})\end{array}$ & $\begin{array}{c}\text { Initial Charge } \\
\text { capacity }(\mathbf{m A h} / \mathbf{g})\end{array}$ & $\begin{array}{c}\text { Coulombic } \\
\text { efficiency (\%) }\end{array}$ \\
\hline $\mathrm{P}-\mathrm{Si}$ & 2746 & 2437 & 88.8 \\
$5 \mathrm{RGO} / \mathrm{Si}$ & 2116 & 2000 & 94.5 \\
$10 \mathrm{RGO} / \mathrm{Si}$ & 2693 & 2645 & 98.2 \\
$15 \mathrm{RGO} / \mathrm{Si}$ & 1745 & 1697 & 97.2 \\
$10 \mathrm{RGO} / \mathrm{Si}-600$ & 2317 & 2160 & 93.2 \\
\hline
\end{tabular}

Table S4. The cycle performance of P-Si and RGO/Si at different current.

\begin{tabular}{|c|c|c|c|c|c|c|}
\hline Electrodes & $\begin{array}{l}0.1 \mathrm{~A} \\
\text { rate }\end{array}$ & $\begin{array}{l}0.3 \mathrm{~A} \\
\text { rate }\end{array}$ & $\begin{array}{c}0.5 \mathrm{~A} \\
\text { rate }\end{array}$ & 1 A rate & $\begin{array}{l}0.1 \mathrm{~A} \\
\text { rate }\end{array}$ & $\begin{array}{l}\text { conservation } \\
\text { rate after } \\
\text { cycle }\end{array}$ \\
\hline $\mathrm{P}-\mathrm{Si}$ & $\begin{array}{c}2747 \\
\mathrm{mAh} / \mathrm{g}\end{array}$ & $\begin{array}{c}2257 \\
\mathrm{mAh} / \mathrm{g}\end{array}$ & $\begin{array}{c}1755 \\
\mathrm{mAh} / \mathrm{g}\end{array}$ & $\begin{array}{c}1302 \\
\mathrm{mAh} / \mathrm{g}\end{array}$ & $\begin{array}{c}1935 \\
\mathrm{mAh} / \mathrm{g}\end{array}$ & $70 \%$ \\
\hline $5 \mathrm{RGO} / \mathrm{Si}$ & $\begin{array}{c}2151 \\
\mathrm{mAh} / \mathrm{g}\end{array}$ & $\begin{array}{c}1368 \\
\mathrm{mAh} / \mathrm{g}\end{array}$ & $\begin{array}{c}1170 \\
\mathrm{mAh} / \mathrm{g}\end{array}$ & $\begin{array}{c}895 \\
\mathrm{mAh} / \mathrm{g}\end{array}$ & $\begin{array}{c}1557 \\
\mathrm{mAh} / \mathrm{g}\end{array}$ & $72 \%$ \\
\hline 10RGO/Si & $\begin{array}{c}2717 \\
\mathrm{mAh} / \mathrm{g}\end{array}$ & $\begin{array}{c}2411 \\
\mathrm{mAh} / \mathrm{g}\end{array}$ & $\begin{array}{c}2086 \\
\mathrm{mAh} / \mathrm{g}\end{array}$ & $\begin{array}{c}1547 \\
\mathrm{mAh} / \mathrm{g}\end{array}$ & $\begin{array}{c}2222 \\
\mathrm{mAh} / \mathrm{g}\end{array}$ & $82 \%$ \\
\hline $15 \mathrm{RGO} / \mathrm{Si}$ & $\begin{array}{c}2053 \\
\mathrm{mAh} / \mathrm{g}\end{array}$ & $\begin{array}{c}1780 \\
\mathrm{mAh} / \mathrm{g}\end{array}$ & $\begin{array}{c}1377 \\
\mathrm{mAh} / \mathrm{g}\end{array}$ & $\begin{array}{c}1053 \\
\mathrm{mAh} / \mathrm{g}\end{array}$ & $\begin{array}{c}1695 \\
\mathrm{mAh} / \mathrm{g}\end{array}$ & $83 \%$ \\
\hline $\begin{array}{c}\text { 10RGO/Si- } \\
600\end{array}$ & $\begin{array}{c}2455 \\
\mathrm{mAh} / \mathrm{g}\end{array}$ & $\begin{array}{c}2241 \\
\mathrm{mAh} / \mathrm{g}\end{array}$ & $\begin{array}{c}2023 \\
\mathrm{mAh} / \mathrm{g}\end{array}$ & $\begin{array}{c}1730 \\
\mathrm{mAh} / \mathrm{g}\end{array}$ & $\begin{array}{c}2156 \\
\mathrm{mAh} / \mathrm{g}\end{array}$ & $88 \%$ \\
\hline
\end{tabular}


Table S5. The electrochemical performance of $10 \mathrm{RGO} / \mathrm{Si}-600$ in this work and references for LIBs

\begin{tabular}{cccc}
\hline Reference & Cycle number & Current density & Specific Capacity \\
\hline This work & 100 & $2 \mathrm{~A} / \mathrm{g}$ & $728 \mathrm{~mA} \mathrm{~h} / \mathrm{g}$ \\
This work & 100 & $0.1 \mathrm{~A} / \mathrm{g}$ & $1969 \mathrm{~mA} \mathrm{~h} / \mathrm{g}$ \\
7 & 130 & $0.1 \mathrm{~A} / \mathrm{g}$ & $1055.1 \mathrm{~mA} \mathrm{~h} / \mathrm{g}$ \\
27 & 30 & $0.1 \mathrm{~A} / \mathrm{g}$ & $1168 \mathrm{~mA} \mathrm{~h} / \mathrm{g}$ \\
28 & 100 & $2.4 \mathrm{~A} / \mathrm{g}$ & $\sim 1000 \mathrm{~mA} \mathrm{~h} / \mathrm{g}$ \\
32 & 100 & $0.05 \mathrm{~A} / \mathrm{g}$ & $\sim 1219 \mathrm{~mA} \mathrm{~h} / \mathrm{g}$ \\
34 & 100 & $0.8 \mathrm{~A} / \mathrm{g}$ & $\sim 880 \mathrm{~mA} \mathrm{~h} / \mathrm{g}$ \\
37 & 50 & $0.5 \mathrm{~A} / \mathrm{g}$ & $1280 \mathrm{~mA} \mathrm{~h} / \mathrm{g}$ \\
38 & 100 & $0.05 \mathrm{~A} / \mathrm{g}$ & $1215 \mathrm{~mA} \mathrm{~h} / \mathrm{g}$ \\
\hline
\end{tabular}

